\title{
HAUSDORFF'S MOMENT SEQUENCES AND EXPONENTIAL CONVOLUTIONS
}

\section{A. Z. Grinshpan}

\begin{abstract}
We introduce some classes of exponential compositions related to Hausdorff's moment sequences. It turns out that one of these classes coincides with the known class of analytic functions defined by the $q$-difference operator. We give sharp bounds for some functionals in the considered classes.
\end{abstract}

\section{Some compositions related to convolutions}

Let $A_{0}$ be the class of analytic functions $h(z), h(0)=0$ in the unit disk $E:|z|<1$, and let $A_{0}^{+}$be the subclass of $A_{0}$ consisting of all functions $h$ with nonnegative coefficients $\{h\}_{n}, n \geq 1$. The notation $\{h\}_{n}$ here and below stands for the $n^{\text {th }}$ Taylor coefficient of a function (or a formal power series) $h$ about zero. For $h \in A_{0}^{+}$, let $G(h)$ be the subclass of functions $\omega \in A_{0}$ which satisfy the condition $\left|\{\omega\}_{n}\right| \leq\{h\}_{n}, n \geq 1$. Note that the symbol « has been used for such a coefficient majorization.

For any two functions $g, \omega \in A_{0}$, let $g * \omega$ denote the function

$$
(g * \omega)(z)=\sum_{n=1}^{\infty}\{g\}_{n}\{\omega\}_{n} z^{n}, \quad z \in E .
$$

Clearly, $g * \omega \in A_{0}$. It is called the Hadamard product of $g$ and $\omega$ or the convolution of $g$ and $\omega$ (see Ruscheweyh's book [14]).

Lemma 1. Let $h_{1}, h_{2} \in A_{0}^{+}$and let $\omega_{1} \in G\left(h_{1}\right), \omega_{2} \in G\left(h_{2}\right)$. Then $\omega_{1} \omega_{2} \in G\left(h_{1} h_{2}\right)$.

Lemma 2. Let $\Phi(z, w)=\sum_{n, k=0}^{\infty} \alpha_{n, k} z^{n} w^{k}$ be a formal power series in two variables with $\alpha_{n, k} \geq 0 \quad(n, k=0,1, \ldots)$, and let $g, h \in A_{0}^{+}$. Then for any $\omega \in G(h)$ and $N=1,2, \ldots$,

$$
\left|\{f\}_{N}\right| \leq\{F\}_{N}
$$

where

$$
f(z)=\Phi(z,(g * \omega)(z))
$$

and

$$
F(z)=\Phi(z,(g * h)(z)) .
$$

The equality in (1) holds for $\{F\}_{N} \neq 0$ iff $\arg \left\{(g * \omega)^{k}\right\}_{N-n}=$ constant and $\left|\left\{(g * \omega)^{k}\right\}_{N-n}\right|=\left\{(g * h)^{k}\right\}_{N-n}$ provided that $\alpha_{n, k}\left\{(g * h)^{k}\right\}_{N-n} \neq 0,0<k+n \leq N$.

Received July 11, 1995, revised September 4, 1995.

1991 Mathematics Subject Classification. Primary 30C99, 30C45, 30E05, 30C50.

Key words and phrases: Hausdorff's moment problem, analytic functions, convolutions, exponential compositions, coefficient bounds, univalent functions. 
Proof. Equation (2) gives

$$
\left|\{f\}_{N}\right| \leq \sum_{n, k=0}^{N} \alpha_{n, k}\left|\left\{(g * \omega)^{k}\right\}_{N-n}\right|, \quad N \geq 1 .
$$

Hence, by Lemma 1, equation (3) and mathematical induction, the proof is completed.

Let us call the function $F$, defined by (3), the envelope function for every nonempty set of functions $f$ defined by (2). In Lemma 3 and below, the notation $M(r, f)$ stands for $\max _{|z|=r}|f(z)|$ where $f \in A_{0}$ and $r \in[0,1)$.

Lemma 3. Let $g, h \in A_{0}^{+}$and $\omega \in G(h)$. Let $\Phi(z, w)=z e^{w}$, and let $f$ and $F$ be defined by (2) and (3), respectively. Then $M(r, f) / F(r)$ and $\left|f\left(r e^{i \theta}\right)\right| / F(r)$ for any $\theta \in[0,2 \pi)$ are non-increasing functions of $r$ in $(0,1)$. If $\left|f\left(r e^{i \theta}\right)\right|=F(r)$ for some $r \in(0,1)$ and $\theta \in[0,2 \pi)$, then $f(z)=e^{i \theta} F\left(e^{-i \theta} z\right)$ for each $z \in E$.

Proof. For the given function $\Phi$, equations (2) and (3) imply that

$$
\frac{d}{d r}\left[\log \frac{\left|f\left(r e^{i \theta}\right)\right|}{F(r)}\right]=\sum_{n=1}^{\infty} n\{g\}_{n} r^{n-1}\left(\operatorname{Re}\left\{\omega e^{i n \theta}\right\}_{n}-\{h\}_{n}\right) \leq 0 .
$$

Hence, $\left|f\left(r e^{i \theta}\right)\right| / F(r)$ is a nonincreasing function of $r$ in $(0,1)$ for any $\theta \in[0,2 \pi)$. If $\left|f\left(r e^{i \theta}\right)\right|=F(r)$ for some $r \in(0,1)$ and $\theta \in[0,2 \pi)$, then for every $n \geq 1$ either $\{\omega\}_{n}=\left\{h\left(e^{-i \theta} z\right)\right\}_{n}$ or $\{g\}_{n}=0$. Therefore, $f(z)=e^{i \theta} F\left(e^{-i \theta} z\right)$ for each $z \in E$.

Let $0<r_{1}<r_{2}<1$ and let $\theta \in[0,2 \pi)$ satisfy the condition $M\left(r_{2}, f\right)=\left|f\left(r_{2} e^{i \theta}\right)\right|$. It follows that

$$
\frac{M\left(r_{1}, f\right)}{F\left(r_{1}\right)} \geq \frac{\left|f\left(r_{1} e^{i \theta}\right)\right|}{F\left(r_{1}\right)} \geq \frac{\left|f\left(r_{2} e^{i \theta}\right)\right|}{F\left(r_{2}\right)}=\frac{M\left(r_{2}, f\right)}{F\left(r_{2}\right)}
$$

See statements similar to Lemma 3 given in Goodman's book [4, Ch. 6] for univalent functions.

\section{Moment sequences and some integral operators}

A sequence of numbers $\left\{\alpha_{n}\right\}_{0}^{\infty}$ is said to be a moment sequence if it is possible to determine a function $\nu(t)$ of bounded variation in the interval $[0,1]$ such that

$$
\alpha_{n}=\int_{0}^{1} t^{n} d \nu(t), \quad n=0,1, \ldots
$$

It is known that a sequence can have at most one representation (4) if $\nu$ is a normalized function of bounded variation. That is,

$$
\nu(0)=0, \quad \nu(t)=\frac{\nu(t+0)+\nu(t-0)}{2}, \quad t \in(0,1) .
$$

Such a normalization of the function $\nu$ does not change the value of the integral (4) (see e.g., [15, Ch. 1,2]).

The statement of Hausdorff's moment problem is as follows: Find conditions such that a sequence of numbers $\left\{\alpha_{n}\right\}_{0}^{\infty}$ is a moment sequence. This problem was solved completely by $\mathrm{F}$. Hausdorff in 1921. The similar problem for nondecreasing and bounded functions $\nu(t)$ in $[0,1]$ also was solved (see [9], [15, Ch. 3]). We formulate these results in Theorems $\mathrm{A}$ and $\mathrm{B}$. 
Theorem A. A necessary and sufficient condition that $\left\{\alpha_{n}\right\}_{0}^{\infty}$ is a moment sequence is that the sequence $\left\{\beta_{n}\right\}_{0}^{\infty}$ with

$$
\beta_{n}=\sum_{k=o}^{n}\left(\begin{array}{l}
n \\
k
\end{array}\right)\left|\sum_{l=0}^{n-k}(-1)^{l}\left(\begin{array}{c}
n-k \\
l
\end{array}\right) \alpha_{n-l}\right|
$$

is bounded.

According to Schur (see [9]), a sequence of nonnegative numbers $\left\{\alpha_{n}\right\}_{m}^{\infty}$ is said to be completely monotonic if it satisfies the condition

$$
\sum_{l=0}^{n}(-1)^{n-l}\left(\begin{array}{l}
n \\
l
\end{array}\right) \alpha_{n-l+k} \geq 0, \quad n=0,1, \ldots, k=m, m+1, \ldots .
$$

Let us call a completely monotonic sequence $\left\{\alpha_{n}\right\}_{m}^{\infty}$ minimal completely monotonic if for any $\varepsilon>0$ the sequence $\left\{\alpha_{n}^{\prime}\right\}_{m}^{\infty}$ where $\alpha_{m}^{\prime}=\alpha_{m}-\varepsilon$ and $\alpha_{n}^{\prime}=\alpha_{n}(n \geq m+1)$ is not completely monotonic. Clearly, for any minimal completely monotonic sequence, $a_{m}=\sup _{n \geq 1} \sum_{l=0}^{n-1}(-1)^{l}\left(\begin{array}{c}n \\ n-1-l\end{array}\right) a_{l+m+1}$.

Theorem B. A necessary and sufficient condition that the sequence $\left\{\alpha_{n}\right\}_{0}^{\infty}$ have the expression

$$
\alpha_{n}=\int_{0}^{1} t^{n} d \nu(t), \quad n=0,1, \ldots,
$$

where $\nu(t)$ is nondecreasing and bounded for $t \in[0,1]$, is that the sequence be completely monotonic.

Theorems A and B allow us to describe the set of analytic functions in the class $A_{0}$ which generate convolutions with the simplest integral representation. It will be sufficient to consider Riemann-Stieltjes integrals on $[\varepsilon, 1], \varepsilon \geq 0$, and some of their limit values. In fact, only integration near zero may be not acceptable in some cases. In these cases we define

$$
\int_{0}^{1} f(x) d \mu(x):=\int_{0+}^{1} f(x) d \mu(x)+\left[\frac{f(x)}{x}\right]_{x=0} \cdot[x \mu(x)]_{x=0}^{x=0^{+}}
$$

provided that: $f(x)$ and $\mu(x)$ are defined for $x \in(0,1],[f(x) / x]$ and $[x \mu(x)]$ are defined at $x=0$, the Riemann-Stieltjes integral of $f(x)$ with respect to $\mu(x)$ from any $\varepsilon \in(0,1)$

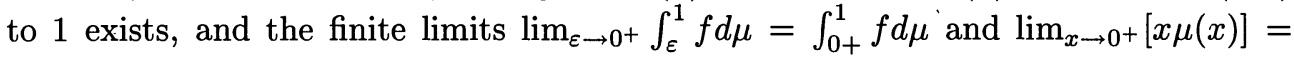
$[x \mu(x)]_{x=0+}$ exist.

We need the following two lemmas.

Lemma 4. (see e.g., [15, Ch. 1]) If $\eta(x)$ and $\varphi(x)$ are continuous and $\nu(x)$ is of bounded variation in $[a, b]$, and if

$$
\mu(x)=-\int_{x}^{b} \varphi(t) d \nu(t), \quad x \in[a, b]
$$

then

$$
\int_{a}^{b} \eta(x) d \mu(x)=\int_{a}^{b} \eta(x) \varphi(x) d \nu(x)
$$

Lemma 5. If $\nu(x)$ is of bounded variation in $[0,1]$, then

$$
\lim _{x \rightarrow 0+} x \int_{x}^{1} \frac{\nu(t)}{t^{2}} d t=\nu\left(0^{+}\right)
$$


Proof. It is sufficient to prove (6) for any function $\nu$ nondecreasing and bounded in $[0,1]$. For such a function $\nu$, any $\varepsilon \in(0,1]$ and $x \in\left(0, \varepsilon^{2}\right)$, let

$$
A(x, \varepsilon)=x \int_{x}^{\varepsilon} \frac{\nu(t)}{t^{2}} d t
$$

We have from (7)

$$
\nu\left(0^{+}\right)(1-\varepsilon) \leq A(x, \varepsilon) \leq \nu(\varepsilon), \quad x \in\left(0, \varepsilon^{2}\right) .
$$

Hence,

$$
\nu\left(0^{+}\right)(1-\varepsilon) \leq \lim _{x \rightarrow 0^{+}} \inf A(x, \varepsilon) \leq \lim _{x \rightarrow 0^{+}} \sup A(x, \varepsilon) \leq \nu(\varepsilon) .
$$

To finish the proof, note that we can replace $A(x, \varepsilon)$ by $A(x, 1)$ in $(8)$, and then let $\varepsilon$ go to $0^{+}$.

Theorem 1. A necessary and sufficient condition that the convolution of a fixed function $g \in A_{0}$ and an arbitrary function $f \in A_{0}$ have the expression

$$
(f * g)(z)=\int_{0}^{1} f(z x) d \mu(x), \quad z \in E,
$$

where

$$
\mu(x)=-\int_{x}^{1} \frac{d \nu(t)}{t}, \quad x \in(0,1], \quad[x \mu(x)]_{x=0}=\nu(0)-\nu(0+),
$$

and $\nu(t)$ is of bounded variation in $[0,1]$, is that the sequence

$$
\left\{\beta_{n}\right\}_{0}^{\infty}, \quad \beta_{n}=\sum_{k=0}^{n}\left(\begin{array}{l}
n \\
k
\end{array}\right)\left|\sum_{l=0}^{n-k}(-1)^{l}\left(\begin{array}{c}
n-k \\
l
\end{array}\right)\{g\}_{n+1-l}\right|
$$

be bounded.

Each function $\mu$ defined by (10) generates such a function $g \in A_{0}$ by (9) with $f(z)=z^{n}, n=1,2, \ldots$.

Proof. Using Theorem A, we conclude that

$$
(f * g)(z)=\sum_{n=1}^{\infty}\{f\}_{n}\{g\}_{n} z^{n}=\int_{0}^{1} f(z x) \frac{d \nu(x)}{x}
$$

for any $f \in A_{0}, z \in E$, and some function $\nu$ of bounded variation in [0,1] iff the sequence (11) is bounded. For any $\varepsilon \in(0,1)$, Lemma 4 with $\eta(x)=f(z x)$ and $\varphi(x)=\frac{1}{x}$ gives

$$
\int_{\varepsilon}^{1} f(z x) \frac{d \nu(x)}{x}=\int_{\varepsilon}^{1} f(z x) d \mu(x)
$$

where $\mu$ is defined by (10).

It follows that (9) with (10) (see definition (5)) and (12) are equivalent if $[x \mu(x)]_{x=0+}$ exists and equals 0 . Using (10) and the formula for integration by parts, we have

$$
x \mu(x)=\nu(x)-x \nu(1)-x \int_{x}^{1} \frac{\nu(t)}{t^{2}} d t, \quad x \in(0,1) .
$$

Hence, Lemma 5 gives

$$
\lim _{x \rightarrow 0+}[x \mu(x)]=0 .
$$

Now let $\mu$ be defined by (10), and let the corresponding function of bounded variation $\nu$ from (10) generate the moment sequence $\alpha_{n}=\int_{0}^{1} t^{n} d \nu(t)(n=0,1, \ldots)$. This 
sequence is bounded, and therefore, the function $g(z)=\sum_{n=1}^{\infty} \alpha_{n-1} z^{n}$ belongs to $A_{0}$. The above argument shows that $g$ satisfies (9).

Remark 1. Note that $\mu(1-x)$ defined by (10) is normalized in $[0,1)$ iff $\nu^{*}(x)=$ $\nu(x)-\nu(0)$ is normalized in $[0,1)$ (see e.g., [15, Ch. 1]). It follows from the proof of Theorem 1 that for every $n=1,2, \ldots$,

$$
\alpha_{n}=\int_{0}^{1} x^{n} d \mu(x)=\int_{0}^{1} x^{n-1} d \nu(x) .
$$

As mentioned above, there is at most one such a function $\nu$ which is of bounded variation and normalized in $[0,1]$.

We conclude that for every admissible function $g \in A_{0}$, there is at most one representation (9) which is valid for all functions $f \in A_{0}$ (or even for a fixed function $f$ with $\{f\}_{n} \neq 0, n \geq 1$ ) if $\mu$ is defined by (10) and

$$
\mu(x)=\frac{\mu(x+0)+\mu(x-0)}{2}, \quad x \in(0,1) .
$$

Indeed, if there exists another function

$$
\tilde{\mu}(x)=-\int_{x}^{1} \frac{d \tilde{\nu}(t)}{t}, \quad x \in(0,1], \quad[x \tilde{\mu}(x)]_{x=0}=-\tilde{\nu}\left(0^{+}\right),
$$

$(\tilde{\nu}(t)$ is a normalized function of bounded variation in $[0,1])$ which satisfies (9) for every $f \in A_{0}$ and $z \in E$, then $\nu(t)-\nu(0)=\tilde{\nu}(t), t \in[0,1]$. Hence, $\tilde{\mu}(x)=\mu(x)$, $x \in(0,1]$, and $[x \tilde{\mu}(x)]_{x=0}=[x \mu(x)]_{x=0}=\nu(0)-\nu(0+)$.

We shall consider a class $\mathcal{M}$ of nondecreasing functions $\mu(x)<\infty$ in $(0,1]$ with $\int_{0}^{1} x d \mu(x)<\infty$. According to the definition (5), for each $\mu \in \mathcal{M}$, the finite limit values $\int_{0+}^{1} x d \mu(x)$ and $[x \mu(x)]_{x=0+}$ exist and $[x \mu(x)]$ is defined at $x=0$. Lemma 6 allows us to define the class $\mathcal{M}$ properly. Also, this lemma and Theorem 2 show why we need an additional restriction at $x=0$.

Lemma 6. Let $\mu(x),|\mu|<\infty$, be nondecreasing in $(0,1]$, and let $\int_{0+}^{1} x d \mu(x)<\infty$. Then the limit value $[x \mu(x)]_{x=0+}$ exists and equals 0 .

Proof. If $\mu(x) \geq 0$ in $(0,1]$, then $0 \leq x \mu(x) \leq x \mu(1)$ in $(0,1]$, and the statement of Lemma 6 follows.

Now let $\mu(x)<0$ for $0<x<b \leq 1$. The formula for integration by parts gives

$$
\int_{\varepsilon}^{b} x d \mu(x)=b \mu(b)-\varepsilon \mu(\varepsilon)-\int_{\varepsilon}^{b} \mu(x) d x
$$

for any $\varepsilon \in(0, b)$. It follows that

$$
\int_{\varepsilon}^{b} \mu(x) d x \geq b \mu(b)-\int_{0+}^{1} x d \mu(x)>-\infty
$$

and therefore, the finite limit value $\int_{0+}^{b} \mu(x) d x$ exists. Hence, the finite limit value $\gamma=[\varepsilon \mu(\varepsilon)]_{\varepsilon=0+}$ exists also. Clearly, $\gamma \leq 0$. If $\gamma<0$, then for some $c \in(0, b)$ and $\alpha \in(\gamma, 0), x \mu(x)<\alpha$ for each $x \in(0, c)$. Again using the formula for integration by parts, we have for any $\varepsilon \in(0, c)$

$$
\int_{\varepsilon}^{c} x d \mu(x)=c \mu(c)-\varepsilon \mu(\varepsilon)-\int_{\varepsilon}^{c} \mu(x) d x>c \mu(c)-\alpha \log \left(\frac{e c}{\varepsilon}\right) .
$$


Hence,

$$
\lim _{\varepsilon \rightarrow 0+}\left[c \mu(c)-\alpha \log \left(\frac{e c}{\varepsilon}\right)\right] \leq \int_{0+}^{1} x d \mu(x)<\infty .
$$

It follows that $\alpha \geq 0$. We have reached a contradiction. Thus, $\gamma=0$.

Now we can give an appropriate definition of the class $\mathcal{M}$. Let $\mathcal{M}$ be the class of nondecreasing functions $\mu(x),|\mu|<\infty$ in $(0,1]$ with $\int_{0+}^{1} x d \mu(x)<\infty$ and a given value $[x \mu(x)]_{x=0} \in(-\infty, 0]$. Let $\mathcal{M}_{0}$ be the subclass of functions $\mu \in \mathcal{M}$ such that $x \mu(x)$ is right-continuous at $x=0$. Lemma 6 shows that $\mathcal{M}_{0}=\left\{\mu \in \mathcal{M}:[x \mu(x)]_{x=0}=0\right\}$.

Theorem 2. A necessary and sufficient condition that the convolution of a fixed function $g \in A_{0}$ and an arbitrary function $f \in A_{0}$ has the expression

$$
(f * g)(z)=\int_{0}^{1} f(z x) d \mu(x), \quad z \in E,
$$

where $\mu \in \mathcal{M}$, is that the sequence $\{g\}_{n}, n \geq 1$ is completely monotonic.

Each function $\mu \in \mathcal{M}$ generates such a function $g \in A_{0}$ by (13) with $f(z)=z^{n}$, $n=1,2, \ldots$

Proof. Theorem B gives that

$$
(f * g)(z)=\sum_{n=1}^{\infty}\{f\}_{n}\{g\}_{n} z^{n}=\int_{0}^{1} f(z x) \frac{d \nu(x)}{x}
$$

for any $f \in A_{0}, z \in E$, and some nondecreasing and bounded function $\nu$ in $[0,1]$ iff the sequence $\{g\}_{n}, n \geq 1$, is completely monotonic.

Let $g \in A_{0}$, and let the sequence $\{g\}_{n}, n \geq 1$ be completely monotonic. Lemmas 4 and 5 show that (14) implies (13) with $\mu$ defined by (10) and $[x \mu(x)]_{x=0+}=0$ as in the proof of Theorem 1. Clearly, this function $\mu$ is nondecreasing and nonpositive in $(0,1]$, and $[x \mu(x)]_{x=0} \in(-\infty, 0]$. Setting $f(z)=z$ in $(13)$, we have

$$
\int_{0}^{1} x d \mu(x)=\{g\}_{1}<\infty
$$

Hence, $\mu \in \mathcal{M}$.

Now let us assume that (13) holds with $g \in A_{0}$ and $\mu \in \mathcal{M}$. Then (15) holds, $[x \mu(x)]_{x=0} \leq 0$, and according to Lemma $6,[x \mu(x)]_{x=0+}=0$.

Let

$$
\nu(t)=-\int_{t}^{1} x d \mu(x), \quad t \in(0,1], \quad \nu(0)=\nu(0+)+[x \mu(x)]_{x=0} .
$$

Equations (15) and (16) give that $\nu(t) \in\left[-\{g\}_{1}+[x \mu(x)]_{x=0}, 0\right]$ and is nondecreasing in $[0,1]$. Let

$$
\begin{aligned}
& \tilde{\mu}(x)=-\int_{x}^{1} \frac{d \nu(t)}{t}, \quad x \in(0,1], \\
& {[x \tilde{\mu}(x)]_{x=0}=\nu(0)-\nu(0+)=[x \mu(x)]_{x=0} .}
\end{aligned}
$$

Using Lemma 4 with $\varphi(t)=t$ and $\eta(t)=1 / t$ in $[x, 1]$, we obtain $\tilde{\mu}(x)=\mu(x)-\mu(1)$, $x \in(0,1]$. Taking into account $(13)$, we have

$$
(f * g)(z)=\int_{0}^{1} f(z x) d \tilde{\mu}(x)
$$

for any $f \in A_{0}$ and $z \in E$. 
Using Lemma 4 again, we show that (18) and (17) with $[x \tilde{\mu}(x)]_{x=0+}=0$ imply (14). Therefore, the sequence $\{g\}_{n}, n \geq 1$ is completely monotonic.

For any function $\mu \in \mathcal{M}$, let $\nu$ be defined by (16). As mentioned above, $\nu$ is nondecreasing and bounded in $[0,1]$. This function generates some moment sequence $\alpha_{n}=\int_{0}^{1} t^{n} d \nu(t), n=0,1, \ldots$, which is completely monotonic by Theorem B. In particular, this sequence is bounded. Therefore, the corresponding function $g(z)=$ $\sum_{n=1}^{\infty} \alpha_{n-1} z^{n}$ belongs to $A_{0}$. The equation (18) with $\tilde{\mu}(x)=\mu(x)-\mu(1)$ defined by (17) and the above argument show that $g$ is generated by $\mu$ as in (13).

Remark 2. According to Remark 1 , for every admissible function $g \in A_{0}$, there is at most one representation (13) which is valid for all functions $f \in A_{0}$ (or even for a fixed function $f$ with $\left.\{f\}_{n} \neq 0, n \geq 1\right)$, if $\mu \in \mathcal{M}$ and $\mu$ satisfies

$$
\mu(x)=\frac{\mu(x+0)+\mu(x-0)}{2}, \quad x \in(0,1), \quad \mu(1)=0 .
$$

Corollary 1. Under the conditions of Theorem 2, $\mu \in \mathcal{M}_{0}$ iff the sequence $\{g\}_{n}$, $n \geq 1$, is minimal completely monotonic.

Proof. Let the sequence $\{g\}_{n}, n \geq 1$, be minimal completely monotonic and $\mu \notin \mathcal{M}_{0}$. This means that $\mu \in \mathcal{M}$ with $[x \mu(x)]_{x=0}<0$. Let $\tilde{\mu}(x)=\mu(x), x \in(0,1]$, and $[x \tilde{\mu}(x)]_{x=0}=0$; then $\tilde{\mu} \in \mathcal{M}$. The function $\tilde{\mu}$ generates a function $\tilde{g} \in A_{0}$ by (13) with $f(z)=z^{n}, n=1,2, \ldots$ We have $\{\tilde{g}\}_{1}=\{g\}_{1}+[x \mu(x)]_{x=0},\{\tilde{g}\}_{n}=\{g\}_{n}, n \geq 2$. Since the sequence $\{\tilde{g}\}_{n}, n \geq 1$, is completely monotonic and $[x \mu(x)]_{x=0}<0$, we have reached a contradiction. Now let us assume that $\mu \in \mathcal{M}_{0}$ and the sequence $\{g\}_{n}$, $n \geq 1$, is not minimal completely monotonic. Without loss of generality, we can assume that $\mu$ is normalized as in Remark 2. Let $g(z)=\tilde{g}(z)+\varepsilon z, \varepsilon>0, z \in E$, where both sequences $\{g\}_{n}$ and $\{\tilde{g}\}_{n}, n \geq 1$, are completely monotonic. According to Theorem 2, $\{\tilde{g}\}_{n}=\int_{0}^{1} x^{n} d \tilde{\mu}(x), n \geq 1$, where $\tilde{\mu} \in \mathcal{M}$ with $[x \tilde{\mu}(x)]_{x=0} \leq 0$. Therefore, $\{g\}_{n}=$ $\int_{0}^{1} x^{n} d \tilde{\tilde{\mu}}(x), n \geq 1$, where $\tilde{\tilde{\mu}}(x)=\mu(x), x \in(0,1]$, and $[x \tilde{\tilde{\mu}}]_{x=0}=[x \tilde{\mu}(x)]_{x=0}-\varepsilon \leq 0$. Since $\tilde{\tilde{\mu}} \in \mathcal{M}$ and $\mu$ is unique, $\tilde{\tilde{\mu}}(x)=\mu(x), x \in(0,1]$, and $[x \tilde{\mu}(x)]_{x=0}=\varepsilon=0$. We have reached a contradiction again.

Every admissible function $g$, either from Theorem 1 or Theorem 2, generates some linear integral operator $I(f): A_{0} \rightarrow A_{0}$. For example, if $g(z)=\log (1 /(1-z))$ and $\mu(x)=\log x \in \mathcal{M}_{0}$ in Theorem 2 , we get the classical operator of Alexander [1]

$$
I(f)(z)=\int_{0}^{z} \frac{f(\zeta)}{\zeta} d \zeta
$$

See Miller and Mocanu [13], for example, for material concerning various generalizations of Alexander's operator.

We shall consider exponential convolutions of the form

$$
f(z)=z \exp \{(g * \omega)(z)\}
$$

where the function $g \in A_{0}^{+}$and has a completely monotonic sequence of Taylor coefficients. Let $\mathcal{E} C_{h}(g)$ be the class of all functions $f$ defined by (20) where $\omega \in G(h)$ for some fixed function $h \in A_{0}^{+}$. According to Theorem 2, for every such pair of functions $g$ and $h$, we have the corresponding exponential integral operator

$$
J(\omega)(z)=z \exp \left\{\int_{0}^{1} \omega(z x) d \mu(x)\right\}: G(h) \rightarrow \mathcal{E} C_{h}(g)
$$

where $\mu \in \mathcal{M}$. 
If a function $\mu \in \mathcal{M}$ with $[x \mu(x)]_{x=0}=-a \leq 0$ related to some admissible function $g$ by (13) is given, then we use the notation $E C_{h}(\mu \mid a)$ for the class $\mathcal{E} C_{h}(g)$. Let $E C_{h}(\mu)=\bigcup_{a \geq 0} E C_{h}(\mu \mid a)$. Clearly, each function $f_{a} \in E C_{h}(\mu \mid a)$ can be represented in the form

$$
f_{a}(z)=f(z) \exp \left\{a\{\omega\}_{1} z\right\}, \quad z \in E,
$$

where $f \in E C_{h}(\mu \mid 0)$ and $\omega \in G(h)$. Thus, the case when $x \mu(x)$ is right-continuous at $x=0$ is the most important one.

The function

$$
F(z)=z \exp \left\{\int_{0}^{1} h(z x) d \mu(x)\right\}
$$

is the envelope function for the class $E C_{h}(\mu)$. Lemmas 2 and 3 give some extremal properties of this function on $E C_{h}(\mu)$.

Let $\mu \in \mathcal{M}$ and

$$
L(\mu)= \begin{cases}0 & \text { if } \int_{0}^{1} x d \mu(x)=0 \\ \infty & \text { if } \int_{0}^{1} x^{2} d \mu(x) \neq 0 \\ 1 & \text { otherwise }\end{cases}
$$

Note that if $L(\mu)<\infty$, then the corresponding function $g(z)$ from (13) is a linear function $a z$ with $a \geq 0$.

Theorem 3. Let $h \in A_{0}^{+}, \mu \in \mathcal{M}$, and let $F$ be defined by (21). Then, for any $f(z)=z \exp \left\{\int_{0}^{1} \omega(x z) d \mu(x)\right\} \in E C_{h}(\mu)$ with $\omega \in G(h)$,

(a) $\left|\{f(z)\}_{N}\right| \leq\{F\}_{N}, N \geq 2$,

(b) $|f(z)| \leq F(|z|), z \in E$.

For some natural $N \geq 2$, let $\{h\}_{n}>0(n=1, \ldots, k)$ where $k=\min \{N-1, L(\mu)\}$ and $L(\mu) \neq 0$ is defined by (22). Then the equality in (a) holds iff $\{\omega\}_{n}=\left\{h\left(z e^{i \theta}\right)\right\}_{n}$ for every $n=1, \ldots, k$ and for some $\theta \in[0,2 \pi)$.

If for some $z=r e^{i \theta} \in E-\{0\}|f(z)|=F(r)$, then for each $z \in E, f(z)=$ $e^{i \theta} F\left(e^{-i \theta} z\right)$.

Proof. According to Theorem 2 and the argument following, every function $f \in$ $E C_{h}(\mu)$ is an exponential convolution of the form (20). We have the inequality (a) from Lemma 2. Let $\varphi(z)=\int_{0}^{1} \omega(x z) d \mu(x), \psi(z)=\int_{0}^{1} h(x z) d \mu(x)$, and let the function $h$ satisfy the additional conditions of Theorem 3 . It follows that the functions $\varphi$ and $\psi$ can be represented in the form

$$
\varphi(z)=\sum_{n=1}^{L} a_{n} z^{n} \quad \text { and } \quad \psi(z)=\sum_{n=1}^{L} b_{n} z^{n}, \quad z \in E,
$$

where $\left|a_{n}\right| \leq b_{n}$ and $b_{n} \neq 0$ for $n=1, \ldots, k, k \leq L=L(\mu)$. We used the definition of completely monotonic sequences.

Lemma 2 gives equality in (a) if and only if

$$
\arg \left\{\varphi^{l}\right\}_{N-1}=\text { constant and }\left|\left\{\varphi^{l}\right\}_{N-1}\right|=\left\{\psi^{l}\right\}_{N-1} \text { for } l=1, \ldots, N-1 .
$$

Let $l=N-1$. Then we get $\left|a_{1}\right|=b_{1}>0$ and

$$
\arg \left\{\varphi^{l}\right\}_{N-1}=(N-1) \arg a_{1}
$$

for $l=1, \ldots, N-1$. The case $k=1$ is trivial. If $k>1$, then for every $l=2, \ldots, N-2$, $\left\{\varphi^{l}\right\}_{N-1}$ is a sum of products of coefficients $a_{1}, a_{2}, \ldots$ and of a positive constant such 
that every product has the same argument $(N-1) \arg a_{1}$. Among all of these products are nonzero terms of the form $(N-n)\left(a_{1}^{N-1-n} \cdot a_{n}\right)$ for $n=2, \ldots, k$. It follows that for every $n=2, \ldots, k,\left|a_{n}\right|=b_{n}>0$ and $\arg \left[(N-n) a_{1}^{N-1-n} a_{n}\right]=(N-1) \arg a_{1}$. Hence, $\arg a_{n}=n \arg a_{1}$ for $n=2, \ldots, k$. Therefore, $\{\omega\}_{n}=\left\{h\left(z e^{i \theta}\right)\right\}_{n}$ for every $n=1, \ldots, k$ and $\theta=\arg \{\omega\}_{1}$.

The inequality (b) and the statement about the equality sign follow from Lemma 3. Also, this inequality follows either from the inequality (a) or directly from the definition of the class $E C_{h}(\mu)$.

Let us consider a simple example showing the importance of the additional conditions in Theorem 3.

Let $\mu(x)=x \in \mathcal{M}_{0}, h(z)=3 z^{2}+4 z^{3} \in A_{0}^{+}$, and $N=6$. For $n=1,2, \ldots$, we have

$$
\int_{0}^{1} x^{n} d \mu(x)=\frac{1}{n+1}
$$

Clearly, $k=5$, but $\{h\}_{1}=\{h\}_{4}=\{h\}_{5}=0$. The representation (21) gives

$$
F(z)=z \exp \left\{z^{2}+z^{3}\right\} \text {. }
$$

Hence, $\{F\}_{6}=1$. Let $\omega(z)=3 e^{2 i \theta} z^{2}+4 e^{i \alpha} z^{3} \in G(h)$ for some $\theta, \alpha \in[0, \pi / 2], \alpha \neq 3 \theta$. Then

$$
f(z)=z \exp \left\{\int_{0}^{1} \omega(x z) d \mu(x)\right\}=z \exp \left\{e^{2 i \theta} z^{2}+e^{i \alpha} z^{3}\right\} \in E C_{h}(x)
$$

and $\left|\{f\}_{6}\right|=\left|e^{i(2 \theta+\alpha)}\right|=\{F\}_{6}$. However, $\{\omega\}_{2}=3 e^{2 i \theta}=\left\{h\left(z e^{i \theta}\right)\right\}_{2}$ and $\{\omega\}_{3}=$ $4 e^{i \alpha} \neq 4 e^{i 3 \theta}=\left\{h\left(z e^{i \theta}\right)\right\}_{3}$.

\section{The classes $E C(\mu)$}

Let $\mu \in \mathcal{M}_{0}$. We denote by $E C(\mu)$ the class of functions

$$
f(z)=z \exp \left\{\int_{0}^{1} \omega(z x) d \mu(x)\right\}
$$

where $\omega \in A_{0}$ and satisfies the condition

$$
\operatorname{Re}\{\omega(z)\}>-\frac{1}{2}, \quad z \in E .
$$

Let $W$ be the class of all such functions $\omega$. Due to the classical result of Caratheodory [2], it follows that for every $\omega \in W\left|\{\omega\}_{n}\right| \leq 1, n \geq 1$, or $\omega \in G\left(\omega^{*}\right)$ where $\omega^{*}(z)=z /(1-z)$. Therefore, $E C(\mu)$ is a subclass of the class $E C_{\omega^{*}}(\mu \mid 0)$.

For any function $\omega \in W$, using the Herglotz representation formula, we have

$$
\omega(z)=\int_{0}^{2 \pi} \omega^{*}\left(e^{i \alpha} z\right) d \nu(\alpha), \quad z \in E
$$

where $d \nu$ is a positive unit measure on $[0,2 \pi]$ (see e.g., $[4$, Ch. 7]).

Equations (23) and (25) imply that for every $f \in E C(\mu)$ and $z \in E$

$$
f(z)=z \exp \left\{\int_{0}^{1} \int_{0}^{2 \pi} w^{*}\left(x e^{i \alpha} z\right) d \nu(\alpha) d \mu(x)\right\} .
$$

The function

$$
F_{\mu}(z)=z \exp \left\{\int_{0}^{1} \omega^{*}(x z) d \mu(x)\right\}
$$

is the envelope function for the class $E C(\mu)$. 
We shall consider three particular classes $E C(\mu)$ and estimate some traditional functionals on the classes $E C(\mu)$ in general.

Let $S$ be the class of univalent functions $f \in A_{0}, f^{\prime}(0)=1$, and let $S^{*}$ be the class of starlike functions $f \in S$.

Theorem 4. $E C\left(\log x^{2}\right)=S^{*}$.

Proof. For every function $f \in E C\left(\log x^{2}\right)$, we have from (23) and (19)

$$
f(z)=z \exp \left\{\int_{0}^{z} \frac{2 \omega(\zeta)}{\zeta} d \zeta\right\}
$$

where $\omega \in W$ and $z \in E$.

Equation (28) gives

$$
\frac{z f^{\prime}(z)}{f(z)}=1+2 \omega(z)
$$

Since $\omega(0)=0$ and $\operatorname{Re}\{1+2 \omega(z)\}>0$, we use Nevanlinna's condition and conclude that $f \in S^{*}$ (see e.g., [4, Ch. 8]). Thus, $E C\left(\log x^{2}\right) \subseteq S^{*}$. These steps can be reversed to show that $S^{*} \subseteq E C\left(\log x^{2}\right)$.

Clearly, $F_{\log x^{2}}(z)$ is the Koebe function $\mathcal{K}(z)=z /(1-z)^{2}$. It is well known that this function realizes the extremal values of $\left|\{f\}_{n}\right| \quad(n \geq 2),|\arg (f(z) / z)|$, and lower and upper bounds of $|f(z)|(z \in E)$ in the class $S^{*}$ (see e.g., [4, Ch. 8]).

Note that if $f\left(r e^{i \theta}\right)=\operatorname{Re}^{i \phi} \in S^{*}$, then $\phi_{\theta} \geq 0$, and hence, $R_{r}=(R / r) \phi_{\theta} \geq 0$. Therefore, for any $q \in(0,1)$, the inequality

$$
\left|\frac{f(q z)}{f(z)}\right| \leq 1, \quad z \in E
$$

holds.

Recently Ismail et al. [10] introduced the class $P S_{q}$ of functions $f \in A_{0}, f^{\prime}(0)=1$, which satisfy the inequality $(29)$ for some fixed $q \in(0,1)$. This class was defined in [10] by the $q$-difference operator

$$
\left(\mathcal{D}_{q} f\right)(z)=\frac{f(z)-f(q z)}{z(1-q)}, \quad z \neq 0, \quad\left(\mathcal{D}_{q} f\right)(0)=f^{\prime}(0)
$$

which plays an important role in the theory of basic hypergeometric series. It was proved in [10] that the basic hypergeometric function of Heine with some normalization belongs to $P S_{q}$.

We show that for every $q \in(0,1)$, the class $P S_{q}$ is one of the classes $E C(\mu)$.

Theorem 5. For every $q \in(0,1), P S_{q}=E C\left(\mu_{q}\right)$ where $\mu_{q} \in \mathcal{M}_{0}$ and is defined by

$$
\mu_{q}(x)=\log q^{2 k}, \quad x \in\left[q^{k}, q^{k-1}\right)
$$

for $k=0,1, \ldots$.

Proof. It is easily verified that $\mu_{q} \in \mathcal{M}_{0}$ for any $q \in(0,1)$. It follows from (30) that for $n=1,2, \ldots$,

$$
\int_{0}^{1} x^{n} d \mu_{q}(x)=2\left(\log \frac{1}{q}\right) \sum_{k=0}^{\infty} q^{k n}=\frac{2 \log \frac{1}{q}}{1-q^{n}} .
$$

Hence, from (23), we have for any $f \in E C\left(\mu_{q}\right)$ and $z \in E$

$$
f(z)=z \exp \left\{2\left(\log \frac{1}{q}\right) \sum_{n=1}^{\infty} \frac{\{\omega\}_{n} z^{n}}{1-q^{n}}\right\}
$$


where $\omega \in W$.

Equation (31) gives

$$
\frac{f(q z)}{f(z)}=q \exp \left\{2(\log q) \sum_{n=1}^{\infty}\{\omega\}_{n} z^{n}\right\}=\exp \{(2 \omega(z)+1) \log q\} .
$$

Therefore, the inequality (29) holds and $f \in P S_{q}$. Thus $E C\left(\mu_{q}\right) \subseteq P S_{q}$. It follows from [10, Lemma 2.2 and Theorem 1.13] that every function $f \in P S_{q}$ has the representation

$$
f(z)=\frac{z}{\prod_{n=0}^{\infty}\left\{h\left(z q^{n}\right) / q\right\}}
$$

where the function $h$ satisfies the conditions: $h-q \in A_{0}, h(z) \neq 0$, and $h(z) \in E$ for any $z \in E$. Here the convergence is uniform on compact subsets of $E$. Since every such function $h$ can be represented in the form $h(z)=q \exp \left\{\log q^{2} \cdot \omega(z)\right\}$ where $\omega \in W$, our steps can be reversed to show that $P S_{q}$ is a subset of $E C\left(\mu_{q}\right)$.

The envelope function $F_{\mu_{q}}$ for the class $E C\left(\mu_{q}\right)$ is

$$
F_{\mu_{q}}(z)=z \exp \left\{2\left(\log \frac{1}{q}\right) \sum_{n=1}^{\infty} \frac{z^{n}}{1-q^{n}}\right\} .
$$

It was proved in [10] that this function maximizes $\left|\{f\}_{n}\right|, n \geq 2$, within the class $P S_{q}$. We extend this result for $P S_{q}$ and the above mentioned results for the class $S^{*}$ for all classes $E C(\mu)$. Also some other extremal properties of the functions $F_{\mu}$ will be given.

Theorem 6. Let $\mu \in \mathcal{M}_{0}, f \in E C(\mu)$, and let $F_{\mu}$ be defined by (27). Then

(a) $\left|\{f\}_{n}\right| \leq\left\{F_{\mu}\right\}_{n}, n \geq 2$,

(b) $M(r, f) / F_{\mu}(r)$ and $\left|f\left(r e^{i \theta}\right)\right| / F_{\mu}(r)$ for any $\theta \in[0,2 \pi)$ are nonincreasing functions of $r$ in $(0,1)$.

If $|z|=r<1$,

(c) $-F_{\mu}(-r) \leq|f(z)| \leq F_{\mu}(r)$,

(d) $\left|\arg \frac{f(z)}{z}\right| \leq \max _{|\zeta|=r}\left|\arg \frac{F_{\mu}(\zeta)}{\zeta}\right|=\max _{\theta \in[0,2 \pi)} \int_{0}^{1} \frac{x r \sin \theta}{1-2 x r \cos \theta+x^{2} r^{2}} d \mu(x)$.

The equality sign in (a) occurs iff

$$
f(z)=e^{-i \theta} F_{\mu}\left(e^{i \theta} z\right)
$$

for some $\theta \in[0,2 \pi)$. The equality $|f(z)|=F_{\mu}(|z|)$ for some $z \in E-\{0\}$ holds iff $f$ is defined by (32) with $\theta=-\arg z$. The equality $|f(z)|=-F_{\mu}(-|z|)$ for some $z \in E-\{0\}$ holds iff $f$ is defined by (32) with $\theta=\pi-\arg z$.

Proof. The statements (a) and (b), and the right-hand inequality in (c) follow from Theorem 3 and Lemma 3. If $\int_{0}^{1} x d \mu(x)=0$, then $f(z)=F_{\mu}(z)=z$. Otherwise, according to Theorem 3, the equality in (a) for some $n \geq 2$ implies that $\left|\{\omega\}_{1}\right|=1$, where $\omega \in W$ is defined by (23). It follows that in this case $\omega(z)=\omega^{*}\left(e^{i \theta} z\right)$ for some $\theta \in[0,2 \pi)$. The representation (25) implies that for each $\omega \in W$

$$
-\frac{|z|}{1+|z|} \leq \operatorname{Re}\{\omega(z)\}, \quad z \in E,
$$

with equality iff $w(z)=w^{*}\left(e^{i \theta} z\right)$ where $\theta=\pi-\arg z$ (see e.g., [4, Ch. 7]). Using this, (23), and (27), we obtain the left-hand inequality in (c). Of course, the right-hand inequality in (c) also can be proved in this way. 
For every $\omega \in W$, we have from (25)

$$
|\operatorname{Im}\{\omega(z)\}| \leq \max _{\alpha \in[0,2 \pi)}\left|\operatorname{Im}\left\{\omega^{*}\left(e^{i \alpha} z\right)\right\}\right| .
$$

Using this inequality, (23), and (27), we get (d).

Corollary 2. The Koebe domain for the class $E C(\mu)$ is the open disk centered at zero with the radius

$$
r(\mu)=\exp \left\{-\int_{0}^{1} \frac{x}{1+x} d \mu(x)\right\} .
$$

In particular, we have the well-known value $r\left(\log x^{2}\right)=1 / 4$ for the class $S^{*}$ and a new result $r\left(\mu_{q}\right)=\exp \left\{2(\log q) \sum_{k=0}^{\infty} \frac{q^{k}}{1+q^{k}}\right\}$ for the class $P S_{q}$ where $\mu_{q}$ is defined by (30).

Remark 3. Note that for all $f \in E C(\mu)$ the radius of starlikeness of $f$ is greater than or equal to that of $F_{\mu}$. Equation (26) gives that the starlike radius $r_{\mu}$ of $E C(\mu)$ either is defined by the transcendental equation $\min _{\theta} \operatorname{Re}\left\{\int_{0}^{1} K\left(x r_{\mu} e^{i \theta}\right) d \mu(x)\right\}=-1$ where $K$ is the Koebe function, or $r_{\mu}=1$. Earlier, the starlike radius of $P S_{q}$ was found in [10].

In fact, the classes $E C(\mu), \mu \in \mathcal{M}_{0}$, give some natural extension of the class $P S_{q}$, which in turn is a $q$-extension of the class $S^{*}$. Some properties of this natural extension are easier to prove than those in the $q$-case. It allows us to establish some new $q$-properties and to prove known ones in a simple way. An approach based on a natural extension that includes a $q$-extension of some mathematical object may be effective in other cases also.

Let $G S$ be the class of bounded functions $f \in A_{0}$ satisfying the conditions: $f^{\prime}(0)=$ $1, f(z) / z \neq 0(z \in E)$, and $\inf \{|w|: w \in \partial f(E)\}>0$. This class was introduced by the author and Saff in [8] without the normalization $f^{\prime}(0)=1$. Some estimates of the functional $\sup _{z \in E}\left|\arg \frac{f(z)}{z}\right|$ on the class $G S$ are given in [8]. These results are related to the problem of estimating the argument of approximate conformal mappings of simply connected domains onto the unit disk. The statements (c) and (d) of Theorem 6 and Theorem 7 provide a new stimulus for this problem.

Theorem 7. Let $\mu \in \mathcal{M}_{0}$, then $E C(\mu) \subset G S$ iff

$$
\int_{0}^{1} \frac{x d \mu(x)}{1-x}<\infty
$$

Proof. Let $f \in E C(\mu)$. The formula (23) gives $f^{\prime}(0)=1$. According to the statement (c) of Theorem 6 and the inequality (33), we have

$$
0<\exp \left\{-\int_{0}^{1} \frac{x d \mu(x)}{1+x}\right\} \leq\left|\frac{f(z)}{z}\right| \leq \exp \left\{\int_{0}^{1} \frac{x d \mu(x)}{1-x}\right\}<\infty
$$

for any $z \in E$. Hence $f \in G S$.

If for some $\mu \in \mathcal{M}_{0}, E C(\mu) \subseteq G S$, then $F_{\mu} \in G S$. Therefore, $\sup _{r \rightarrow 1^{-}}\left(\log F_{\mu}(r)\right)=$ $\int_{0}^{1}(x d \mu(x) /(1-x))<\infty$. Note that for $f \in E C(\mu),(33)$ gives

$$
\left|\arg \frac{f(z)}{z}\right| \leq \int_{0}^{1} \frac{x}{1-x} d \mu(x)<\infty, \quad z \in E .
$$

Hence, any function $f \in G S$ with $\sup _{Z \in E}\left|\arg \frac{f(z)}{z}\right|=\infty$ (see [8]) cannot belong to $E C(\mu)$ with condition (33). Thus $E C(\mu) \subset G S$. 


\section{Estimating some logarithmic means on the classes $E C(\mu)$}

For every function $f \in S$, its logarithmic coefficients $\gamma_{n}$ are defined by

$$
\log \frac{f(z)}{z}=\sum_{n=1}^{\infty} 2 \gamma_{n} z^{n}
$$

For each natural $n$, a real nonzero vector $X_{n}=\left(x_{1}, \ldots, x_{n}\right)$ is said to be admissible if the Koebe function maximizes the functional

$$
\mathbf{M}_{X_{n}}(f)=\sum_{k=1}^{n} x_{k}\left(k\left|\gamma_{k}\right|^{2}-\frac{1}{k}\right)
$$

on the class $S$. Thus, for every admissible vector $X_{n}$ and $f \in S$

$$
\mathbf{M}_{X_{n}}(f) \leq 0 \text {. }
$$

Some admissible vectors are very important in the theory of univalent functions. It is well-known that Milin [11] conjectured and de Branges [3] proved that all vectors $\tilde{X}_{n}=(n, n-1, \ldots, 1), n \geq 1$, are admissible. Also, it is known that at the present time this is the only approach to prove the famous Bieberbach conjecture for the Taylor coefficients of univalent functions. We note that, in fact, $\lim _{n \rightarrow \infty} \mathbf{M}_{\tilde{X}_{n}}(f)=-\infty$ for every function $f \in S$ unless $f$ is the Koebe function or one of its rotations [5]. It is proved in [12] that each admissible vector $X_{n}=\left(x_{1}, \ldots, x_{n}\right)$ necessarily satisfies the condition

$$
\min _{\theta \in[0, \pi]} \sum_{k=1}^{n} x_{k} \sin (k \theta)=0 .
$$

For example, the unit vectors $X_{n}^{\prime}=(1,1, \ldots, 1), n \geq 2$, do not satisfy (37). Thus they are not admissible, but $\sup _{S} \mathbf{M}_{X_{n}^{\prime}}(f) \leq \delta<0.312$ where $\delta$ is Milin's constant [11].

For $n \leq 2$ the condition (37) describes the admissible vectors, but it is not sufficient for describing admissible vectors in the general case $[6,7]$. It is stated in $[6,7]$ that the inequality (36) holds for each function $f \in S^{*}$ and for any real nonzero vector $X_{n}$ satisfying (37). In this case only, the Koebe function and its rotations give the equality in (36). Theorem 8 gives an extension of the last statement for all classes $E C(\mu)$.

Clearly, every function $f \in E C(\mu)$ has the only zero at $z=0$. Hence, the function $\log \frac{f(z)}{z}$ is analytic in $E$. Let $f \in E C(\mu)$ and

$$
\log \frac{f(z)}{z}=\sum_{n=1}^{\infty} \beta_{n} z^{n}, \quad z \in E .
$$

Theorem 8. Let $\mu \in \mathcal{M}_{0}$ and for some real numbers $x_{k}, k=1, \ldots, n$

$$
\min _{\theta \in[0, \pi]} \sum_{k=1}^{n} x_{k}\left(k \int_{0}^{1} x^{k} d \mu(x)\right)^{2} \sin k \theta=0 .
$$

For each $f \in E C(\mu)$, let

$$
\mathbf{M}(f)=\sum_{k=1}^{n} x_{k} k\left|\beta_{k}\right|^{2}
$$

where the logarithmic coefficients $\beta_{k}$ are defined by (38).

Finally, let $F_{\mu}$ be defined by (27). Then $F_{\mu}$ maximizes the functional $\mathbf{M}(f)$ on the class $E C(\mu)$. If the sum in (39) is not identically zero, then only the function $F_{\mu}$ and its rotations maximize this functional on $E C(\mu)$. 
Proof. Let $f \in E C(\mu)$. The representation (26) gives

$$
\log \frac{f(z)}{z}=\int_{0}^{1} \int_{0}^{2 \pi} \frac{e^{i \alpha} x z}{1-e^{i \alpha} x z} d \nu(\alpha) d \mu(x)
$$

where $d \nu$ is a positive unit measure.

Using (38), (40), and (41), we obtain

$$
\mathbf{M}(f)=\int_{0}^{2 \pi} \int_{0}^{2 \pi} \sum_{k=1}^{n} x_{k} k\left(\int_{0}^{1} x^{k} d \nu(x)\right)^{2} \cos (k(\alpha-\theta)) d \nu(\alpha) d \nu(\theta) .
$$

The formula (27) gives

$$
\mathbf{M}\left(F_{\mu}\right)=\sum_{k=1}^{n} x_{k} k\left(\int_{0}^{1} x^{k} d \mu(x)\right)^{2}
$$

We show that

$$
\varphi(t)=\sum_{k=1}^{n} x_{k} k\left(\int_{0}^{1} x^{k} d \mu(x)\right)^{2}(1-\cos (k t)) \geq 0
$$

for $|t| \leq 2 \pi$.

Note that $\varphi(-t)=\varphi(2 \pi-t)=\varphi(t)$. Thus, it is enough to prove (44) for $t \in[0, \pi]$. We have $\varphi(0)=0$ and according to $(39), \varphi^{\prime}(t)$ is nonnegative in $[0, \pi]$. The relations (41)-(43) imply the statement of Theorem 8 about extremality of the function $F_{\mu}$. We have that $\mathbf{M}(f)=\mathbf{M}\left(F_{\mu}\right)$ iff

$$
\int_{0}^{2 \pi} \int_{0}^{2 \pi} \varphi(\alpha-\theta) d \nu(\alpha) d \nu(\theta)=0
$$

It follows that if the sum in (39) is not identically zero, then (45) holds iff $\nu$ is a point mass.

Note that if $\mu(x)=\log x^{2}$, then (39) coincides with (37).

Acknowledgment. The author wishes to express his gratitude to A. W. Goodman and M. E. H. Ismail for several helpful discussions.

\section{References}

1. J. W. Alexander, Functions which map the interior of the unit circle upon simple regions, Ann. of Math. 17 (1915), 12-22.

2. C. Caratheodory, Über den Variabilitätsbereich der Fourierschen Konstanten von positiven harmonischen Functionen, Rend. Circ. Mat. Palermo, 32 (1911), 193-217.

3. L. de Branges, A proof of the Bieberbach conjecture, Acta Math. 154 (1985), 137-152.

4. A. W. Goodman, Univalent Functions, V.1, Polygonal Publ. House, Washington, NJ, 1983.

5. A. Z. Grinshpan, Logarithmic coefficients of functions in the class S, Sibirsk. Mat. Zh. 13 (1972), 1145-1157 (in Russian); English transl.: Siberian Math. J. 13 (1972), 793-801.

6. - Method of exponentiation for univalent functions. In: Theory of Functions and Approximations, Izdat. Saratov Univ., Saratov, 1990, Part 2, pp.72-74 (in Russian).

7. __ Advanced coefficient problems for univalent functions. In: Linear and Complex Analysis Problem Book 3, Springer-Verlag, Berlin, 1994, Part 2, pp.397-400.

8. A. Z. Grinshpan and E. B. Saff, Estimating the argument of approximate conformal mappings, Complex Variables 26 (1994), 191-202.

9. F. Hausdorff, Summationmethoden and Momentfolgen, I, Math. Zeitschrift 9 (1921), 74-109.

10. M. E. H. Ismail, E. Merkes, and D. Styer, A generalization of starlike functions, Complex Variables 14 (1990), 77-84.

11. I. M. Milin, Univalent Functions and Orthonormal Systems, Nauka, Moscow, 1971 (in Russian); English transl.: American Math. Soc., Providence, 1977. 
12. I. M. Milin and A. Z. Grinshpan, Logarithmic coefficients means of univalent functions, Complex Variables 7 (1986), 139-147.

13. S. S. Miller and P. T. Mocanu, Classes of univalent integral operators, J. Math. Anal. Appl. 157 (1991), 147-165.

14. St. Ruscheweyh, Convolutions in Geometric Function Theory, Les Presses de L'Université de Montréal, 1982.

15. D. V. Widder, The Laplace Transform, Princeton, Princeton University Press, 1946.

Department of Mathematics, University of South Florida, Tampa, FL 33620, USA

E-mail: azg@math.usf.edu 